\title{
Paraplegia
}

\section{Prediction of Ramp Traversability for Wheelchair Dependent Individuals}

\author{
A. Cappozzo, ${ }^{1}$ F. Felici' ${ }^{1}$ F. Figura' ${ }^{1}$ M. Marchetti, ${ }^{1}$ B. Ricci ${ }^{2}$ \\ ${ }^{1}$ Institute of Human Physiology, Faculty of Medicine and Surgery, Rome University, \\ 'La Sapienza', Visiting Research Professor, Faculty of Medicine and Surgery, Rome \\ University, 'La Sapienza', Italy and Professor Emeritus, University of Massachusetts, \\ Amherst, MA, 01003 USA.
}

\begin{abstract}
Summary
The purpose of this research was to obtain a single objective criterion that would constitute a reliable prediction of length and grade limits for ramps that can be traversed by any special category of wheelchair dependent individuals (WDI). The maximal voluntary force $(M V C)$ is the main limiting factor of the performance of WDI, thus the force required to traverse a ramp has been established by means of a simple mechanical model. The real time course of force application during ascent was experimentally obtained. Then, a simplified law of force application was introduced in a computer simulation program of kinetics and kinematics of ascent. Inputs to the model were also the mass of the subject plus wheelchair, the initial velocity of the chair, and the ramp length. The output of the program was the force requirement for any given length/grade. When capsizing conditions were encountered the simulation ended. We tested the results thus obtained having some selected WDI successfully traversing ramps with the predicted length/grade specifications.
\end{abstract}

Key words: Ramp length/grade predictions; Wheelchair dependent individuals.

Ramps, or inclines, are commonly used to provide opportunities for wheelchair dependent individuals (WDI) to overcome differences between grade levels. Yet, the limits of allowable grades have not been based upon stated scientific criteria. This is reflected in the widely differing standards among various countries For example, the accepted standards vary from $5 \%$ in France and Belgium to $12.5 \%$ in Poland (ICTA, 1974). A survey of the literature reveals one article dealing with ramp grades (Goldsmith, 1976). In that study, a small sample of WDI were requested to ascend ramps of varying grades, and the maximum grades that subjects had traversed without discomfort were judged acceptable.

The present research was aimed at obtaining a single, objective criterion, based on easily-obtainable and reliable measurements, which permits the prediction of allowable limits of length and grade for ramps that can be adopted for any special 
categories of WDI. The maximum voluntary force (MVC) that a subject can exert in pushing a wheel rim is the most important determinant of the limits of possible ascent. Our basic hypothesis was that MVC can be assumed to be a sufficient predictor of WDI ascent capability.

\section{Methods}

The following experimental plan, based on the above stated hypothesis, was adopted:

1. The basic modality of the WDI wheel rim push during ramp ascent was established. Knowledge of the time course of force application on the wheel rim was a necessary premise for the next step.

2. A mathematical simulation of kinetics and kinematics of WDI ramp ascent was incorporated into a computer program. The inputs into this model were the MVC plus the mass of subject + wheelchair while the output was length and grade of the ramp which the subject could be expected to traverse. The necessity of avoiding capsizing by the WDI was also incorporated into the program.

3. The predictive data produced by the model were tested by measuring MVC on WDI, and requiring them to ascend the predicted length/grade.

Symbols and definitions used in this paper:

$\mathrm{m}=$ mass of subject plus wheelchair.

$\ddot{\mathrm{x}}=$ acceleration of subject plus wheelchair centre of gravity along direction of ascent.

$\alpha=$ ramp grade.

$\mathrm{g}=$ gravitational acceleration.

$l=$ ramp length.

$\mathrm{V} 0=$ initial velocity (prior to ascent).

$\sigma=$ range of angular wheel revolution during which the pushing force was applied.

$\mathrm{Fp}=$ pushing force.

$\mathrm{Fpt}=$ pushing force threshold.

$\mathrm{Cf} 1, \mathrm{Cf} 2=$ Friction couple on the rear and front wheels.

$\mathrm{r} 1, \mathrm{r} 2=$ radium of the rear and front wheels

$\mathrm{H} 1, \mathrm{~V} 1, \mathrm{H} 2, \mathrm{~V} 2=$ horizontal and vertical component of reaction forces acting on rear and front wheels.

\section{Mathematical model}

With reference to Figure 1, the following forces were considered: the pushing force exerted by the subject on the wheel $\operatorname{rim}(\mathrm{Fp})$, the inertia force $(\mathrm{m} \ddot{\mathbf{x}})$, the gravitational force $(\mathrm{m} \mathrm{g})$, and the friction force for rear $(\mathrm{Cf} 1 / \mathrm{rl})$ and front $(\mathrm{Cf} 2 / \mathrm{r} 2)$ wheel, according to the following equation (see Appendix I):

1. Fp-Cf1/r1-Cf $2 / \mathrm{r} 2-\mathrm{m}$ g $\sin \alpha-\mathrm{m} \ddot{\mathrm{x}}=0$

Velocity and displacement of the wheelchair were obtained by first and second intregration, respectively, of the above equation.

The capsizing condition was described by the following disequality equation (see Appendix II): 


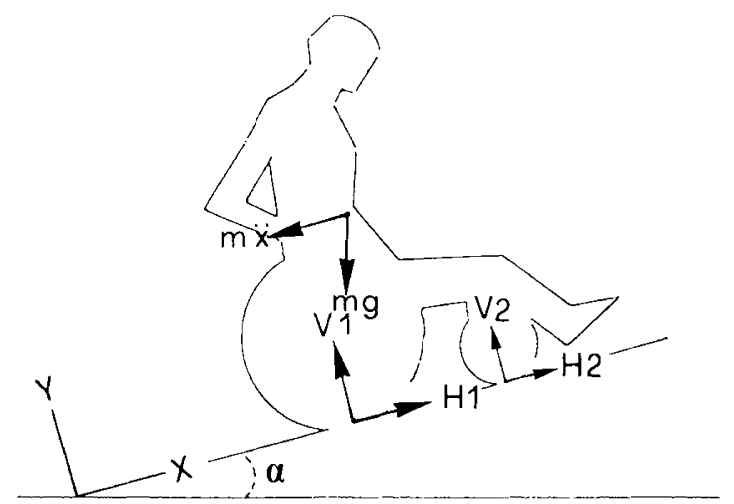

Figure 1 Mechanical model of the system WDI + wheelchair.

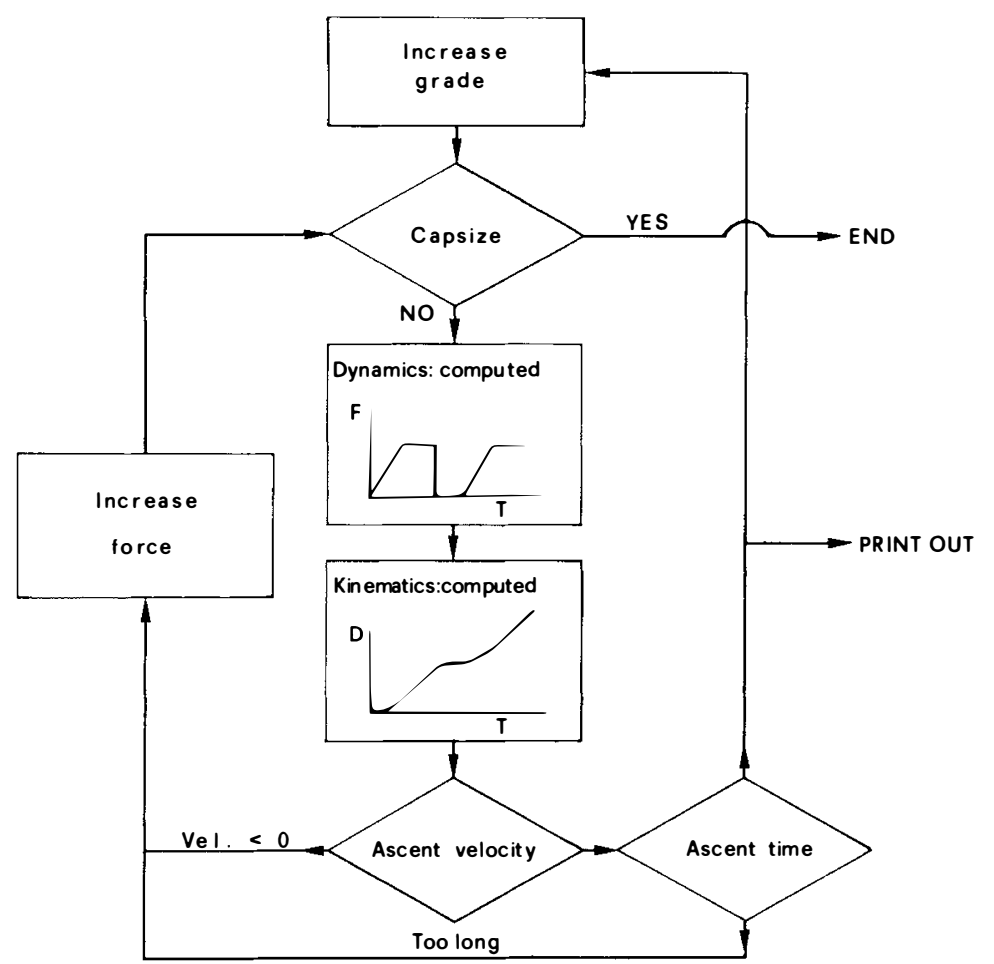

Figure 2 Flow chart of the model adopted for studying the mechanics of ramp ascent (see text for comments).

2. $\ddot{\mathrm{x}}>\mathrm{g} \cos \alpha\left(\mathrm{x}-\mathrm{x}^{\prime}\right) /\left(\mathrm{y}-\mathrm{y}^{\prime}\right)-\mathrm{g} \sin \alpha$ where $x$ and $y$ were the values of $x$ and $y$ co-ordinates of the centre of gravity of the system WDI plus wheelchair, and x' and y' were the co-ordinate of the point of contact of the wheel with pavement.

A flow chart of the program which we adopted for the above mechanical model is presented in Figure 2. The MVC was an input variable, and the constraints were 
that velocity could not be negative and the total time of ascent could not exceed a preselected maximum (Tmax). At the beginning of the calculations a minimum value of grade and Fp were adopted. Running the program, the following possibilities were considered:

(a) Velocity of ascent was negative; Fp was incremented by a finite quantity and ascent started again.

(b) The total time of ascent exceeded the Tmax; Fp was increased by a finite quantity and the ascent started again.

(c) The ascent of the ramp was completed and the grade was incremented by a finite quantity and ascent started again.

(d) When capsizing conditions were encountered, the program stopped.

(e) The Fpt coincided with the selected amount of MVC; the program indicated the maximum grade the subject was able to manage.

In order to identify the model parameters the following quantities had to be experimentally measured on WDI subjects during typical ramp ascents.

(a) Kinematics of CG.

(b) Hand kinematics.

(c) Time history of the pushing force applied by WDI on the wheel rim.

(d) Friction forces.

(e) Location of CG.

(f) MVC of subjects.

(g) Resistance to fatigue in order to establish the percentage of MVC that can be actually employed for ascending selected length/grade ramps.

\section{Subjects}

Twelve WDI volunteered for this study. All had become paraplegic as a consequence of traumatic events, notably vehicular accidents; all were well trained WDI athletes. Of the $12 \mathrm{WDI}, 5$ were selected as being representative of different levels of spinal lesions for kinetic and kinematic measurements (Table I).

\section{CG kinematics}

An adjustable grade ramp (length $6 \mathrm{~m}$, width $1.5 \mathrm{~m}$ ) was specially constructed. Subjects used their own wheelchairs. An accelerometer was applied horizontally on

Table I Characteristics of subjects. C: Cervical; D: Dorsal; L: Lumbar. Numbers indicate the affected spinal segment

\begin{tabular}{lllll}
\hline Sex & $\begin{array}{l}\text { Age } \\
(\mathrm{yr})\end{array}$ & $\begin{array}{l}\text { Spinal } \\
\text { lesion } \\
\text { level }\end{array}$ & $\begin{array}{l}\text { Height } \\
(\mathbf{m})\end{array}$ & $\begin{array}{l}\text { Body } \\
\text { mass } \\
(\mathbf{k g})\end{array}$ \\
\hline M & 29 & D4 & 1.87 & 88 \\
M & 24 & L1 & 1.70 & 68 \\
M & 24 & D12 & 1.75 & 78 \\
M & 34 & C6 & 1.65 & 59 \\
M & 41 & C6 & 1.75 & 78 \\
\hline
\end{tabular}


the midpoint of the wheelchair axle. In this manner, accelerations of CG along the direction of movement during the ascent of the ramp were measured and signals, fed into a personal computer (Olivetti Ml/24), were integrated to obtain velocity and displacement.

\section{Kinematics measurements}

The kinematics of wheelchair performance was also measured by means of a television system. At regulary timed intervals, planar co-ordinates of the centre of the wheel were digitized and its time history thus reconstructed during the ascent of the ramp.

With the television system, the kinematics of the arms in the sagittal plane was also evaluated through the placement of markers on wrist, elbow and shoulder. On the recording was also noted the general posture assumed by WDI during the ascent.

\section{Fp time history}

Using accelerometric data, the typical time course of the force applied by the subject on the wheel rim was calculated as indicated by equation 1 . In order to test the accuracy of the force measurement obtained by accelerometric technique, we instrumented a wheelchair with the dynamometric apparatus previously described (Cerquiglini et al., 1981). In three subjects the ascent of various ramp inclines was performed while measuring $\mathrm{Fp}$ simultaneously with both accelerometric and dynamometric techniques.

\section{Friction force}

Estimation of the friction force of the rotating wheel was accomplished by pulling the chair + WDI at level grade, on the standard pavement, very slowly at constant velocity, with a rope instrumented with a dynamometer (Kistler, mod. 9203).

\section{Centre of gravity identification}

Each subject, sitting in his own wheelchair, was placed on a force plate (Kistler, mod. 9261a) thus allowing for the measurement of the antero-posterior co-ordinate at the point of vertical projection of CG on the platform. During the test, subjects maintained a fixed position with respect to the wheelchair thus allowing for rigid body analysis. To obtain the co-ordinates in the sagittal plane, the mechanical system, consisting of subject plus wheelchair, was inclined at two different angles with respect to the force plate. For each position assumed, a photograph was taken and the ground reaction forces were recorded. Two corresponding force vectors acting on the force plate were thus obtained. By superimposing on the WDI photographic silhouette the aforementioned force vectors, their intersecting point identified the coordinates of the body center of gravity in the sagittal plane. 


\section{MCV measurement}

Force expressed during isometric pushing on the wheel rim was measured by having the wheelchair secured to a wall through a chain arrangement in which a piezoelectric force transducer (Kistler model 9203) was incorporated. Subjects were instructed to exert their MVC by pushing on both wheel rims simultaneously. Three different positions of the hands on the wheel rim were investigated: (a) grasp at the top of the wheel $\left(0^{\circ}\right)$; (b) grasp $30^{\circ}$ forward with respect to the wheel centre $\left(+30^{\circ}\right)$ and (c) grasp $30^{\circ}$ rear with respect to the wheel center $\left(-30^{\circ}\right)$.

For each subject, MVC was fed into the model and the corresponding maximum approachable length/grade thus estimated was actually attempted.

\section{Resistance to fatigue}

Fatigue was estimated through the use of a dynamic test which consisted of a sequence of weight liftings. The weight was suspended over a pulley and connected to the wheelchair. The WDI, while exerting force on the wheel rims, displaced the chair until the weight was lifted for $1 \mathrm{~m}$, then, interrupting the lifting, consented the falling weight to replace the chair in the original position. Forward and backward chair movements were alternated at a cadence of 30 per minute. The weight was chosen to provide resistance equal to 95,85 and $75 \%$ of the WDI maximal isometric force and the test duration was determined by the subject's inability to continue the exercise.

\section{Results}

The reliability of the accelerometric methods for obtaining the pushing force of the subject during the ramp ascent was demonstrated by the comparison with direct dynamometric readings. From the accelerometric and combined video recordings the following relevant common features can be described. Quite the same modality of pushing was observed in each of the 5 male subjects tested. Irrespective of ramp grade, each subject consistently used the same amount of force which varied between 75 to $85 \%$ of MVC. When grade was augmented, the mean speed of progression and the time interval between pushings decreased. Furthermore, the ascent of the ramp was performed with the trunk bent forward and the hands moving consistently from $-30^{\circ}$ to $+30^{\circ}$ with respect to the wheel centre.

The resistance test produced the following results: $95 \%$ MVC could be maintained for no more than 3 to 4 pushing cycles. Cycles of pushes at $85 \%$ of MVC, interspersed by 1 second intervals, were maintained for a period longer than 10 seconds. When the percentage of MVC was reduced to $75 \%$, 1 minute of exercise was comfortably performed.

Position of the CG was noted in the three selected postures. Trunk bending during ascent was observed to be more limited in the cervical spine injured WDI. Therefore this subject presented the rearmost location of CG thus presenting the greater probability for capsizing. In the following calculation we adopted, for sake of safety, the CG coordinates observed in the cervically injured WDI which corresponded to $0.2 \mathrm{~m}$ for anteroposterior coordinate and $0.6 \mathrm{~m}$ for vertical co-ordinate, with respect to the point of contact of the rear wheel with the surface. 


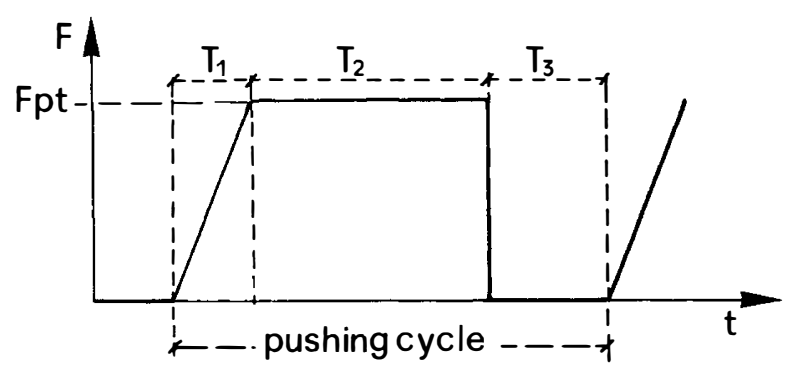

Figure 3 Time course of pushing force $(F)$ applied to wheel rim, according to the mechanical model. $\mathrm{T} 1, \mathrm{~T} 2$ and $\mathrm{T} 3$ indicate the three phases of the cycle, as explained in the text. Fpt is the preselected pushing force threshold.

\section{Model implementation}

The friction force of the rotating wheel was considered negligible. Indeed, it resulted in the order of $1-2 \mathrm{~N}$, and thus less than $1 / 10$ of other considered forces when a ramp grade greater than $3 \%$ was considered.

The time course of Fp was modelled as shown in Figure 3 which was a simple approximation of the modality of pushing we had earlier registered. A single cycle of pushing on the wheel rim was assumed to consist of three phases (T1, T2, T3). During $\mathrm{T} 1$, force was linearly increased from 0 until a pushing force threshold value (Fpt), with a time constant of $2 \mathrm{Fpt}$ per second, which was selected as a best approximation of our experimental data. During T2, constant force (Fpt) was applied until the wheel completed a 1 radian rotation $(\sigma)$; this value of $\sigma$ was selected in accordance with experimental data. During T3, force was null because of the backward movement of the hands to the initial position on the wheel rim. The T3 duration was fixed at $0.4 \mathrm{~s}$, which was the duration we had measured for this movement during fast ascent. Modifications of this law of force application, aimed at achieving a better imitation of natural conditions, failed to show any significant variation of the results provided by the model. Thus, we chose to adopt this simple model to simulate the pushing force applied by the subject on the wheel rim.

The amount of MVC to be employed was selected as a function of the length of the ramp to be traversed and of the time allowed to accomplish that. According to the fatigue measurements, $95 \%$ MVC was chosen for 1 metre length ramp; $85 \%$ MVC for 3 metre length ramp, and $75 \%$ MVC for 6 to 10 metre length ramps. In fact, this adheres to the well established concept that the maximum force available during muscular contraction is inversely proportional to the velocity and duration of movement.

\section{Model validation}

The validity and reliability of the above mentioned model was confirmed by having the subjects achieve the ramp length/grade as predicted by the model on the basis of MVC measurements. Results are reported in Figure 4a in which the data predicted by the model was compared with the ramp and grade that the subject was 

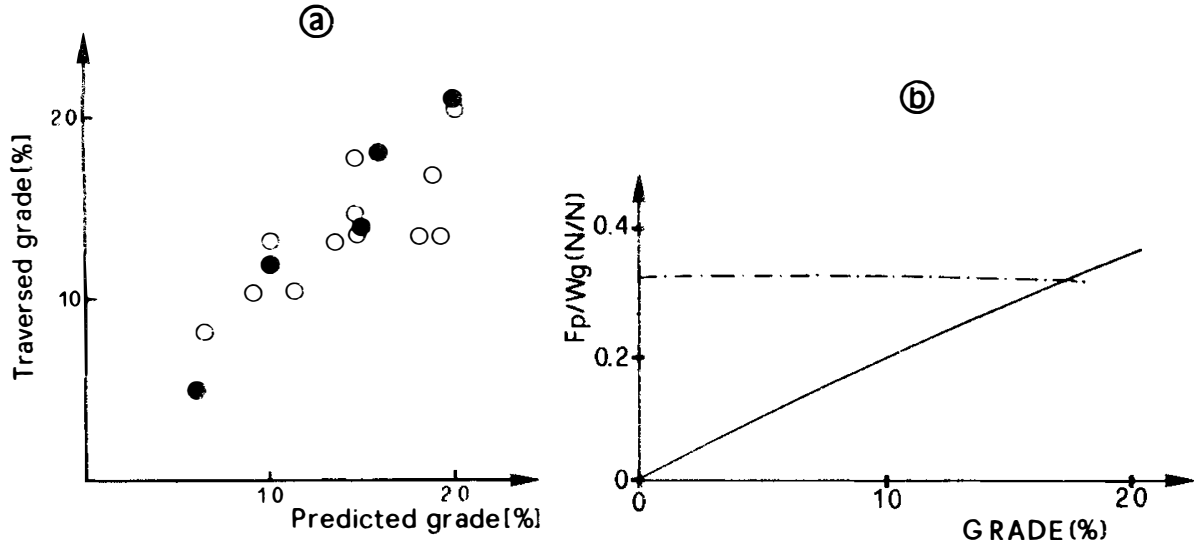

Figure 4 (a): Upper limit of grade traversability as predicted by the model output (abscissa) vs ramps actually traversed by WDI. Open circles indicate 3 metre ramp length; closed circles 6 metre. (b): Force required for traversing the selected ramp length. Force is indicated as a ratio between pushing force $(\mathrm{Fp})$ and weight of subiect + wheelchair $(\mathrm{Wg})$. Dotted line indicates capsizing safety limits.

able to overcome. Only the length which WDI traversed comfortably was considered here.

\section{Conclusion}

A very simple development of the program enabled us to obtain the prediction graphic of Figure $4 \mathrm{~b}$ in which the incline traversability is shown as a function of $\mathrm{Fp} / \mathrm{wg}$, where wg is the. weight of the subject plus wheelchair. Note that in accordance with the above statement on fatigability, the MVC must exceed the required Fp by a percentage which depends on the ramp length. Thus for a 1 metre length, this percentage must be at least $5 \%$, for a 3 metre length $18 \%$, and for 6 to 10 metre length $33 \%$. In any case, our results regarding the capsizing probability suggest the maxima that cannot be exceeded. The dotted line in the Fig. $4 \mathrm{~b}$ indicates such a safety limit. In order to exploit the possibility of our prediction model for architectural designers, further inquiry must be planned in which a subpopulation, statistically representative of the different categories of WDI, shall be submitted to a simple MVC test.

\section{Acknowledgements}

Support for this research was provided by a grant from Regione Lazio, Rome, Italy.

\section{Appendix I}

With reference to the system WDI plus wheelchair, depicted in Figure 1, the dynamic equilibrium to translation entails that:

1. $\mathrm{H} 1+\mathrm{H} 2=\mathrm{m} g \sin \alpha+\mathrm{m} \ddot{\mathrm{x}}$

along the $\mathrm{Y}$ axis, and

2. $\mathrm{V} 1+\mathrm{V} 2=\mathrm{m} \mathrm{g} \cos \alpha$

along the $\mathrm{X}$ axis. 
The dynamic equilibrium to the rotation of rear and front wheel respectively is given by the following equations:

3. $\mathrm{Hl} \mathrm{rl}-\mathrm{Fp} \mathrm{rl}-\mathrm{Cfl}=0$

4. $\mathrm{H} 2 \mathrm{r} 2-\mathrm{Cf} 2=0$

where $\mathrm{r} 1$ and $\mathrm{Cf} 1$ are radius and friction couple for rear wheel and $\mathrm{r} 2$ and $\mathrm{Cf} 2$ are radius and friction couple for front wheel (the inertia couple acting on the wheels is considered to be negligible). Substituting in 1) $\mathrm{H} 1$ as defined in 3) and $\mathrm{H} 2$ as defined in 4) we obtain

5. Fp $-\mathrm{Cf} 1 / \mathrm{r} 1-\mathrm{Cf} 2 / \mathrm{r} 2-\mathrm{mg} \sin \alpha-\mathrm{m} \ddot{\mathrm{x}}=0$

\section{Appendix II}

Capsizing will be encountered when the moments that external forces exert with respect to the point of contact of rear wheel with pavement (having $x^{\prime}$ and $y^{\prime}$ coordinates) are in accordance with the following disequalities:

1. $(\mathrm{m} \ddot{\mathrm{x}}+\mathrm{m} \mathrm{g} \sin \alpha)\left(\mathrm{y}-\mathrm{y}^{\prime}\right)>\mathrm{m} \mathrm{g} \cos \alpha\left(\mathrm{x}-\mathrm{x}^{\prime}\right)$

where $\mathrm{x}$ and $\mathrm{y}$ are the CG coordinate.

1. can be rewritten as

2. $\ddot{\mathrm{x}}>\mathrm{g} \cos \alpha\left(\mathrm{x}-\mathrm{x}^{\prime}\right) /\left(\mathrm{y}-\mathrm{y}^{\prime}\right)-\mathrm{g} \sin \alpha$

\section{References}

Cerquiglini S, Figura F, MARChetTi M, RicCi B 1981 Biomechanics of wheelchair propulsion. In: Morecki A, Fiolelus K, Keolzioz K, Wit A. Biomechanics VII-A 410-419.

ENGEL P, HildEBRANDT G 1974 Wheel chair design technological and physiological aspects. Proceedings of the Royal Society of Medicine 19:409-413.

Hildebrandt G, Voight ED, Bahn D, Berenders B, Kroger J 1970 Energy cost of propelling wheel chair at various speeds: cardiac response and effect on steering accuracy. Archives of Physical Medicine and Rehabilitation 51:131-136.

ICTA 1974 Accessibility for disabled persons - norms in different countries. Compiled by the International Center on Technical Aids, Housing and Transportation, Stockholm, Sweden.

LEHMAN FJ, WARREN CG, HALAR E, STONEBRIDGE JB 1974 Wheel chair propulsion in the quadriplegic patient. Archives of Physical Medicine and Rehabilitation 55:183-186.

MCLAURIN CA, BRUBAKER CE 1979 “Wheel chair biomechanics." Disability, The MacMillan Press 471-477.

SANDERSON DJ, SOMmER HJ 1985 Kinematic features of wheelchair propulsion. Fournal of Biomechanics 18:423-429.

Sawka MN, GlaSer RM, LaUbach LL, Al-Samkari O, Suryaprasad AG 1981 Wheelchair exercise performance of the young, middle-aged, and elderly. Fournal of Applied Physiology 50:824-828.

SAWKa MN, GlaSER RM, WILDE SW, vON LUhrTe TC 1980 Metabolic and circulatory responses to wheelchair and arm crank exercise. Fournal of Applied Physiology 49:784-788.

Stoвoy HB, WilsON-Rich W, LEE M 1971 Workload and energy expenditure during wheel chair propelling. Pareplegia 8:223-230.

VAN DeR WOUde LHV, HeNDRICH KMM, VeEger HEJ et al. 1988 Manual wheelchair propulsion: effect of power output on physiology and technique. Medicine in science and sports 20:70-78.

Van Der Woude LHV, De Groote G, Hollander AP, van IngEN SChenau GJ, Rozendal RH 1986 Wheelchair ergonomics and physiological testing of prototypes. Ergonomics 29:1561-1573. 